\title{
Wave Behaviors of Kundu-Mukherjee-Naskar Model Arising In Optical Fiber Communication Systems with Complex Structure
}

Hadi Rezazadeh

Amol University of Special Modern Technologies

Ali Kurt ( $\square$ alikurt@mku.edu.tr )

Mustafa Kemal Universitesi

Ali Tozar

Mustafa Kemal Universitesi: Hatay Mustafa Kemal Universitesi

Orkun Tasbozan

Hatay Mustafa Kemal Universitesi

Seyed Mehdi Mirhosseini-Alizamini

Payame Noor University

\section{Original Research}

Keywords: KMN model, functional variable method, Rogue waves

Posted Date: February 2nd, 2021

DOI: https://doi.org/10.21203/rs.3.rs-168692/v1

License: (c) (i) This work is licensed under a Creative Commons Attribution 4.0 International License. Read Full License 


\title{
Wave Behaviors of Kundu-Mukherjee-Naskar Model Arising In Optical Fiber Communication Systems with Complex Structure
}

\author{
Hadi Rezazadeh ${ }^{1}$, Ali Kurt ${ }^{2, *}$, Ali Tozar ${ }^{3}$, Orkun Tasbozan ${ }^{4}$, Seyed Mehdi Mirhosseini-Alizamini ${ }^{5}$ \\ ${ }^{\text {I}}$ Faculty of Engineering Technology, Amol University of Special Modern Technologies, \\ Amol, Iran. \\ ${ }^{2}$ Department of Mathematics, Pamukkale University, Denizli, Turkey. \\ ${ }^{3}$ Department of Physics, Hatay Mustafa Kemal University, Hatay, Turkey. \\ ${ }^{4}$ Department of Mathematics, Hatay Mustafa Kemal University, Hatay, Turkey \\ ${ }^{5}$ Department of Mathematics, Payame Noor University (PNU), P.O. Box 19395-3697, \\ Tehran, Iran
}

\begin{abstract}
Rogue waves are very mysterious and extra ordinary waves. They appear suddenly even in a calm sea and are hard to be predicted. Although nonlinear Schrödinger equation (NLS) provides a perspective, it alone can neither detect rogue waves nor provide a complete solution to problems. Therefore, some approximations are still mandatory for both obtaining an exact solution and predicting rogue waves. Such as Kundu-MukherjeeNaskar (KMN) model which allows obtaining lump-soliton solutions considered as rogue waves. In this study the functional variable method is utilized to obtain the analytical solutions of KMN model that corresponds to the propagation of soliton dynamics in optical fiber communication system.
\end{abstract}

Keywords: KMN model, functional variable method, Rogue waves.

\section{Introduction}

Wave motion is very predictable in basic level and can be explained very deterministic way. However, it becomes much more complicated with taking the nonlinear interactions into account. This type of systems called nonlinear or dynamical systems. Although, there has been some development in the mathematical field to understand the behavior of a nonlinear system we are still far from predicting the behavior of such systems deterministically. The first studies on this issue date back almost 150 years to Riemann and stokes. And the studies are continuing with increasing importance and interest [4-6].

The basic idea is concentrated on two types of wave behaviors which are hyperbolic [1] and dispersive. Hyperbolic wave behavior can be formulated mathematically in terms of hyperbolic partial differential equations. Klein-Gordon [13] is a prototype for hyperbolic wave equation. Dispersive waves cannot be characterized easily. Nonhyperbolic waves generally categorized as dispersive waves. However, classification is made on the type of solution rather than on the equations. Korteweg-DeVries equation [14] can be a good example for dispersive waves. There are many equations developed for determining the wave behavior of a dynamical system [7-24].

For a small intersection of a spatiotemporal system the dynamics can be assumed as linear. However, they must be evaluated in terms nonlinear dynamics due to significant modulation of the wave amplitude originated from cumulative nonlinear interactions. Nonlinear

\footnotetext{
${ }^{*}$ Corresponding author:

E-mail address

rezazadehadi1363@gmail.com (Hadi Rezazadeh), pau.dr.alikurt@gmail.com (Ali Kurt),

tozarali@gmail.com (Ali Tozar),

m_mirhosseini@pnu.ac.ir (Seyed Mehdi Mirhosseini-Alizamini).
} 
Schrödinger (NLS) equation is a very common equation which is providing a canonical design of involucre dynamics of a quasi-monochromatic planar wave propagating in a weakly nonlinear dispersive medium when dissipative effects are insignificant [25,26,27].

NLS is employed for many situational models such as propagation of a wave in a Kerr type [28,29] or non- Kerr type [2] medium. Most of them are not fully integrable which means exact solutions cannot be obtained directly. Only approximate numerical solutions with no stable solitons can be obtained [30,31]. Approximations cannot predict rogue waves which can be defined as "localized and isolated surface waves, apparently appear from nowhere, make a sudden hole in the sea just before attaining surprisingly high amplitude and disappear again without a trace" KMN [3]. They proposed a model to by extension of NLS to have an integrable form which allows lump-soliton can be considered as rogue wave model;

$$
i q_{t}=d_{1} q_{x x}-d_{2} q_{y y}+2 i q\left(\sqrt{d_{1}} j^{x}-\sqrt{d_{2}} j^{y}\right), j^{a} \equiv q q_{a}^{*}-q^{*} q_{a} .
$$

And then, they replaced the conventional amplitude-like nonlinear term with the a currentlike nonlinear term which allows them to obtain a fully integrable form of NLS;

$$
i q_{t}+q_{x y}+2 i q\left(q q_{x}^{*}-q^{*} q_{x}\right)=0 .
$$

In this study the wave solutions of KMN model

$$
i q_{t}+\alpha q_{x y}+i \beta q\left(q q_{x}^{*}-q^{*} q_{x}\right)=0 .
$$

which describes the propagation of soliton dynamics in optical fiber communication system. Y1ldırım [35] obtained dark, bright and singular solitons by using trial equation technique for KMN model. Rivzi et al. [36] used csch method, extended Tanh-Coth method and extended rational sinh-cosh method to get the exact solutions of KMN model. Talarposhti et al. [37] employed Exp-function method to yield the optical soliton solutions of considered KMN model.

This work is structured as follows: In Section 2, mathematical analysis of KMN model is given. In Section3, we demonstrate the structure of the functional variable method. In Section 4, we apply this method to find some wave solutions of the equation written above. In Section 5, we give the results and discussion, Section 6 gives the conclusion of the whole research.

\section{Mathematical Analysis}

In order to get started, the following hypothesis is selected:

$$
q(x, y, t)=P(\eta) \exp [i \Phi(x, y, t)],
$$

where $\mathrm{P}(\xi)$ represents the amplitude portion and

$$
\xi=\chi_{1} x+\chi_{2} y-\sigma t,
$$

and the phase portion of the soliton is defined as

$$
\Phi(x, y, t)=-\vartheta_{1} x-\vartheta_{2} y+\varpi t+\theta_{0} .
$$

Here, $\vartheta_{1}$ and $\vartheta_{2}$ are the frequencies of the soliton in the $x$-and $y$-directions respectively while $\varpi$ is the wave number of the soliton and finally $\theta_{0}$ is the phase constant. Also, the parameters $\chi_{1}$ and $\chi_{2}$ in (2.2) represent the inverse width of the soliton along $x$-and $y$ directions respectively, while $\sigma$ represents the velocity of the soliton. Inserting (2.1) along with (2.2) and (2.3) into (1.1) and decomposing into real and imaginary parts, the following pair of equations, respectively yield

$$
\begin{aligned}
& \alpha \chi_{1} \chi_{2} P^{\prime \prime}-\left(\omega+\alpha \vartheta_{1} \vartheta_{2}\right) P-2 \beta \vartheta_{1} P^{3}=0, \\
& \sigma=-\alpha\left(\vartheta_{1} \chi_{2}+\vartheta_{2} \chi_{1}\right) .
\end{aligned}
$$

Eq.(2.4) is transformed into the following one 


$$
P^{\prime \prime}=\frac{\omega+\alpha \vartheta_{1} \vartheta_{2}}{\alpha \chi_{1} \chi_{2}} P+\frac{2 \beta \vartheta_{1}}{\alpha \chi_{1} \chi_{2}} P^{3}
$$

\section{The functional variable method}

This section presents the brief descriptions of the functional variable method $[32,33,34]$.

Suppose that a the NLEE, say in two independent variables to $x$ and $t$ is given by

$$
G\left(u, u_{t}, u_{x x}, \ldots,\right)=0 \text {, }
$$

where $G$ is a function of $u, u_{t}, u_{x x}, \ldots$ and the subscripts denote the partial derivatives of $u(x, t)$ with respect to $x$ and $t$.

A transformation $u(x, t)=U(\eta), \eta=x-\sigma t$ converts the NLEE (3.1) to a nonlinear ODE

$$
F\left(U, U_{\eta}, U_{\eta \eta}, \ldots,\right)=0,
$$

where $F$ is a function of $U, U_{\eta}, U_{\eta \eta}, \ldots$ and its derivatives point out the ordinary derivatives with respect to $\eta$ and where and $\sigma$ is constant to be determine.

Then we make a transformation in which the unknown function $U$ is considered as a functional variable in the form:

$$
U_{\eta}=\Omega(U)
$$

and some successive derivatives of $U$ are

$$
\begin{aligned}
& U_{\eta \eta}=\frac{1}{2}\left(\Omega^{2}\right)^{\prime}, \\
& U_{\eta \eta \eta}=\frac{1}{2}\left(\Omega^{2}\right)^{\prime \prime} \sqrt{\Omega^{2}}, \\
& U_{\eta \eta \eta \eta}=\frac{1}{2}\left[\left(\Omega^{2}\right)^{\prime \prime \prime} \Omega^{2}+\left(\Omega^{2}\right)^{\prime \prime}\left(\Omega^{2}\right)^{\prime}\right],
\end{aligned}
$$

where " " " stands for $\frac{d}{d U}$.

The ODE (3.2) can be reduced in terms of $U, F$ and its derivatives upon using the expressions of Eq. (3.4) into Eq. (3.2) gives

$$
H\left(U, \Omega, \Omega^{\prime}, \Omega^{\prime \prime}, \ldots\right)=0 .
$$

by integrating of Eq. (3.5), Eq. (3.5) can be written with respect to $H$, and it is found the appropriate solutions by using Eq. (3.3) for the investigated problem.

\section{Solutions to the equation (1)}

In this Section we obtain wave solutions of the KMN model by using the functional variable method described in Section 3.

Following Eq. (3.4), it is easy to deduce from Eq. (2.6) an expression for the function $\Omega(U)$

$$
\frac{1}{2}\left(\Omega^{2}\right)^{\prime}=\frac{\omega+\alpha \vartheta_{1} \vartheta_{2}}{\alpha \chi_{1} \chi_{2}} P+\frac{2 \beta \vartheta_{1}}{\alpha \chi_{1} \chi_{2}} P^{3} .
$$

Integrating Eq. (4.1) and setting the constant of integration to $\Xi$ yields

$$
\Omega^{2}=\frac{\omega+\alpha \vartheta_{1} \vartheta_{2}}{\alpha \chi_{1} \chi_{2}} P^{2}+\frac{\beta \vartheta_{1}}{\alpha \chi_{1} \chi_{2}} P^{4}+\Xi,
$$

or

$$
\Omega=P_{\eta}= \pm \sqrt{\frac{\omega+\alpha \vartheta_{1} \vartheta_{2}}{\alpha \chi_{1} \chi_{2}} P^{2}+\frac{\beta \vartheta_{1}}{\alpha \chi_{1} \chi_{2}} P^{4}+\Xi} .
$$

Using Eqs. (2.1), (2.2) and (2.3), we obtain the following wave solutions of the KMN model 
If $\frac{\omega+\alpha \vartheta_{1} \vartheta_{2}}{\alpha \chi_{1} \chi_{2}}>0, \frac{\beta \vartheta_{1}}{\alpha \chi_{1} \chi_{2}}<0$ and $\Xi=0$, we obtain the following bright soliton solutions

$$
\begin{aligned}
q_{1}^{ \pm}(x, y, t)= \pm \sqrt{-\frac{\omega+\alpha \vartheta_{1} \vartheta_{2}}{\beta \vartheta_{1}}} \operatorname{sech}\left(\sqrt{\frac{\omega+\alpha \vartheta_{1} \vartheta_{2}}{\alpha \chi_{1} \chi_{2}}}(\right. & \left.\left.\chi_{1} x+\chi_{2} y+\left(\alpha\left(\vartheta_{1} \eta_{2}+\vartheta_{2} \eta_{1}\right)\right) t\right)\right) \\
& \times \exp \left[i\left(-\vartheta_{1} x-\vartheta_{2} y+\varpi t+\theta_{0}\right)\right] .
\end{aligned}
$$

If $\frac{\omega+\alpha \vartheta_{1} \vartheta_{2}}{\alpha \chi_{1} \chi_{2}}>0, \frac{\beta \vartheta_{1}}{\alpha \chi_{1} \chi_{2}}<0$ and $\Xi=0$, we obtain the following singular soliton solutions

$$
\begin{array}{r}
q_{2}^{ \pm}(x, y, t)= \pm \sqrt{\frac{\omega+\alpha \vartheta_{1} \vartheta_{2}}{\beta \vartheta_{1}}} \operatorname{csch}\left(\sqrt{\frac{\omega+\alpha \vartheta_{1} \vartheta_{2}}{\alpha \chi_{1} \chi_{2}}}\left(\chi_{1} x+\chi_{2} y+\left(\alpha\left(\vartheta_{1} \eta_{2}+\vartheta_{2} \eta_{1}\right)\right) t\right)\right) \\
\times \exp \left[i\left(-\vartheta_{1} x-\vartheta_{2} y+\varpi t+\theta_{0}\right)\right] .
\end{array}
$$

If $\frac{\omega+\alpha \vartheta_{1} \vartheta_{2}}{\alpha \chi_{1} \chi_{2}}<0, \frac{\beta \vartheta_{1}}{\alpha \chi_{1} \chi_{2}}>0$ and $\Xi=0$, we obtain the following periodic wave solutions

$$
\begin{aligned}
& q_{3}^{ \pm}(x, y, t)= \pm \sqrt{-\frac{\omega+\alpha \vartheta_{1} \vartheta_{2}}{\beta \vartheta_{1}}} \sec \left(\sqrt{-\frac{\omega+\alpha \vartheta_{1} \vartheta_{2}}{\alpha \chi_{1} \chi_{2}}}\left(\chi_{1} x+\chi_{2} y+\left(\alpha\left(\vartheta_{1} \eta_{2}+\vartheta_{2} \eta_{1}\right)\right) t\right)\right) \\
& \times \exp \left[i\left(-\vartheta_{1} x-\vartheta_{2} y+\varpi t+\theta_{0}\right)\right], \\
& \begin{aligned}
q_{4}^{ \pm}(x, y, t)= \pm \sqrt{-\frac{\omega+\alpha \vartheta_{1} \vartheta_{2}}{\beta \vartheta_{1}}} \csc \left(\sqrt{-\frac{\omega+\alpha \vartheta_{1} \vartheta_{2}}{\alpha \chi_{1} \chi_{2}}}(\right. & \left.\left.\chi_{1} x+\chi_{2} y+\left(\alpha\left(\vartheta_{1} \eta_{2}+\vartheta_{2} \eta_{1}\right)\right) t\right)\right) \\
& \times \exp \left[i\left(-\vartheta_{1} x-\vartheta_{2} y+\varpi t+\theta_{0}\right)\right] .
\end{aligned}
\end{aligned}
$$

If $\frac{\omega+\alpha \vartheta_{1} \vartheta_{2}}{\alpha \chi_{1} \chi_{2}}<0, \frac{\beta \vartheta_{1}}{\alpha \chi_{1} \chi_{2}}>0$ and $\Xi=-\frac{\left(\omega+\alpha \vartheta_{1} \vartheta_{2}\right)^{2}}{4 \alpha \beta \vartheta_{1} \chi_{1} \chi_{2}}$, we obtain the following dark soliton solution

$$
\begin{array}{r}
q_{5}^{ \pm}(x, y, t)= \pm \sqrt{-\frac{\omega+\alpha \vartheta_{1} \vartheta_{2}}{2 \beta \vartheta_{1}}} \tanh \left(\sqrt{-\frac{\omega+\alpha \vartheta_{1} \vartheta_{2}}{2 \alpha \chi_{1} \chi_{2}}}\left(\chi_{1} x+\chi_{2} y+\left(\alpha\left(\vartheta_{1} \eta_{2}+\vartheta_{2} \eta_{1}\right)\right) t\right)\right) \\
\times \exp \left[i\left(-\vartheta_{1} x-\vartheta_{2} y+\varpi t+\theta_{0}\right)\right] .
\end{array}
$$

If $\frac{\omega+\alpha \vartheta_{1} \vartheta_{2}}{\alpha \chi_{1} \chi_{2}}<0, \frac{\beta \vartheta_{1}}{\alpha \chi_{1} \chi_{2}}>0$ and $\Xi=-\frac{\left(\omega+\alpha \vartheta_{1} \vartheta_{2}\right)^{2}}{4 \alpha \beta \vartheta_{1} \chi_{1} \chi_{2}}$, we obtain the following singular dark soliton solutions

$$
\begin{array}{r}
q_{6}^{ \pm}(x, y, t)= \pm \sqrt{-\frac{\omega+\alpha \vartheta_{1} \vartheta_{2}}{2 \beta \vartheta_{1}}} \operatorname{coth}\left(\sqrt{-\frac{\omega+\alpha \vartheta_{1} \vartheta_{2}}{2 \alpha \chi_{1} \chi_{2}}}\left(\chi_{1} x+\chi_{2} y+\left(\alpha\left(\vartheta_{1} \eta_{2}+\vartheta_{2} \eta_{1}\right)\right) t\right)\right) \\
\times \exp \left[i\left(-\vartheta_{1} x-\vartheta_{2} y+\varpi t+\theta_{0}\right)\right] .
\end{array}
$$

If $\frac{\omega+\alpha \vartheta_{1} \vartheta_{2}}{\alpha \chi_{1} \chi_{2}}>0, \frac{\beta \vartheta_{1}}{\alpha \chi_{1} \chi_{2}}>0$ and $\Xi=-\frac{\left(\omega+\alpha \vartheta_{1} \vartheta_{2}\right)^{2}}{4 \alpha \beta \vartheta_{1} \chi_{1} \chi_{2}}$, we obtain the following periodic wave solutions

$$
\begin{aligned}
q_{7}^{ \pm}(x, y, t)= \pm \sqrt{\frac{\omega+\alpha \vartheta_{1} \vartheta_{2}}{2 \beta \vartheta_{1}}} \tan \left(\sqrt { \frac { \omega + \alpha \vartheta _ { 1 } \vartheta _ { 2 } } { 2 \alpha \chi _ { 1 } \chi _ { 2 } } } \left(\chi_{1} x\right.\right. & \left.\left.+\chi_{2} y+\left(\alpha\left(\vartheta_{1} \eta_{2}+\vartheta_{2} \eta_{1}\right)\right) t\right)\right) \\
& \times \exp \left[i\left(-\vartheta_{1} x-\vartheta_{2} y+\varpi t+\theta_{0}\right)\right],
\end{aligned}
$$




$$
\begin{aligned}
q_{8}^{ \pm}(x, y, t)= \pm \sqrt{\frac{\omega+\alpha \vartheta_{1} \vartheta_{2}}{2 \beta \vartheta_{1}}} \cot \left(\sqrt { \frac { \omega + \alpha \vartheta _ { 1 } \vartheta _ { 2 } } { 2 \alpha \chi _ { 1 } \chi _ { 2 } } } \left(\chi_{1} x\right.\right. & \left.\left.+\chi_{2} y+\left(\alpha\left(\vartheta_{1} \eta_{2}+\vartheta_{2} \eta_{1}\right)\right) t\right)\right) \\
& \times \exp \left[i\left(-\vartheta_{1} x-\vartheta_{2} y+\varpi t+\theta_{0}\right)\right] .
\end{aligned}
$$

\section{Results and discussion}

Figure 1 shows the graphs obtained from the space-time mapping of the solution $\mathrm{q}_{1}$. It can be seen from the figure that the waves have a spatiotemporally extended homoclinic breather wave structure. In this respect, it can be concluded that this $\mathrm{q}_{1}$ solution can be useful in examining the dynamic behavior of rogue waves. It can also be seen that breather waves extend periodically along with time while extending at a certain angle with the $\mathrm{X}$-axis spatially.

Figure 2 shows the graphs obtained from the space-time mapping of the solution $\mathrm{q}_{4}$. Interestingly, although this solution seems to be the solution of heteroclinic waves at first glance, a careful look reveals the difference of the situation. This solution shows the existence of periodically extended homoclinic waves both spatially and temporally.

Figure 2 shows the graphs obtained from the space-time mapping of the solution $\mathrm{q}_{5}$. From this solution, the existence of singular waves extended in time can be seen.

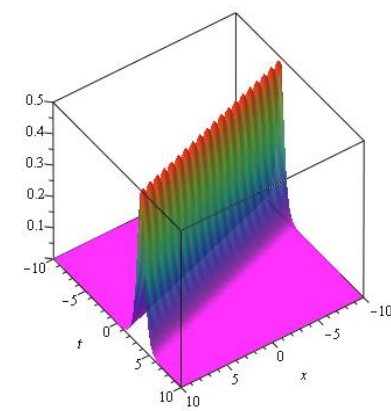

$\left|q_{1}^{+}\right|$

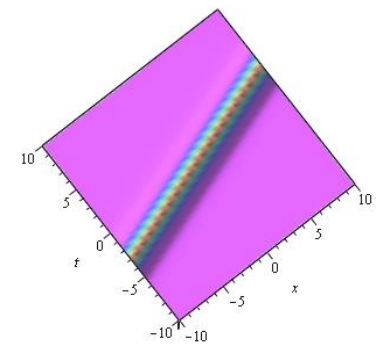

$\left|q_{1}^{+}\right|$

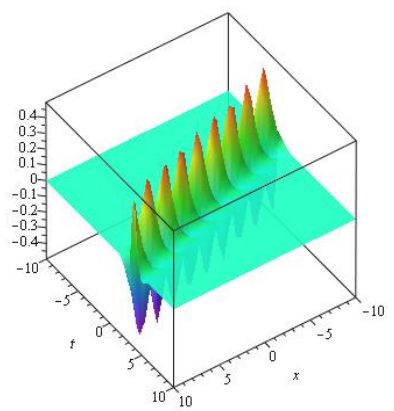

$\operatorname{Re}\left(q_{1}^{+}\right)$

(a)

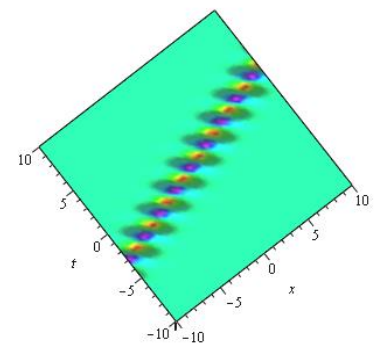

$\operatorname{Re}\left(q_{1}^{+}\right)$

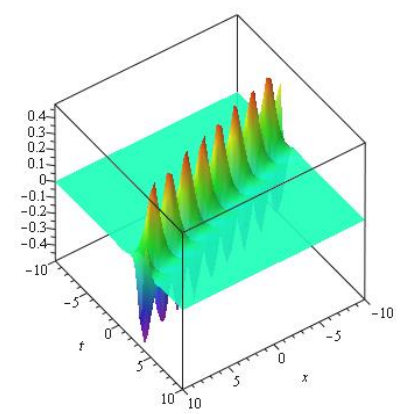

$\operatorname{Im}\left(q_{1}^{+}\right)$

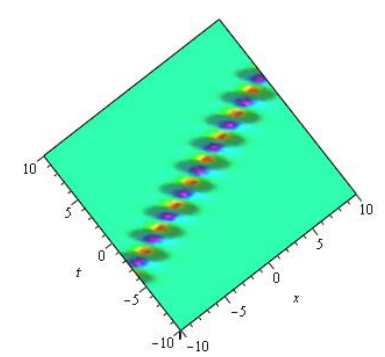

$\operatorname{Im}\left(q_{1}^{+}\right)$

(b) 

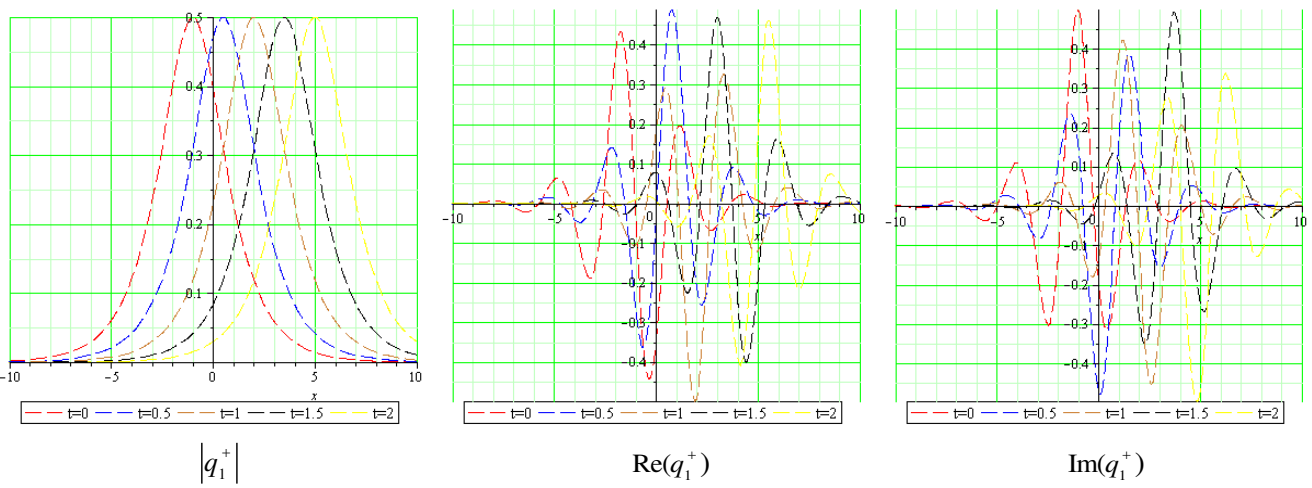

(c)

Fig 1. (a) 3D-plot for $q_{1}^{+}$(b) the contour plot for $q_{1}^{+}$(c) 2D-plot for $q_{1}^{+}$at $\mathrm{t}=0, \mathrm{t}=0.5, \mathrm{t}=1, \mathrm{t}=1.5, \mathrm{t}=2$ .respectively, when $\omega=3, \alpha=1, \vartheta_{1}=-2, \vartheta_{2}=1, \beta=2, \chi_{1}=1, \chi_{2}=1, \eta_{1}=1, \eta_{2}=2, \varpi=2, \theta_{0}=1$ and $y=0.5$.

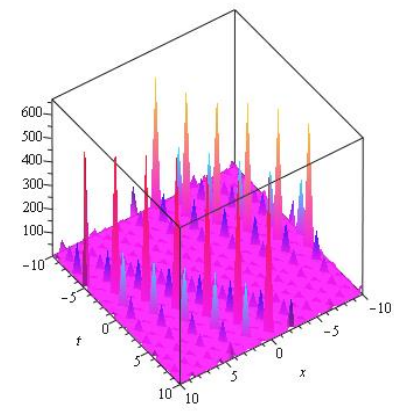

$\left|q_{4}^{-}\right|$

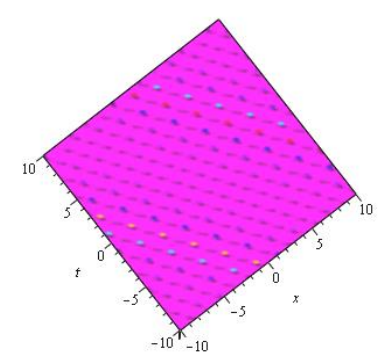

$\left|q_{4}^{-}\right|$

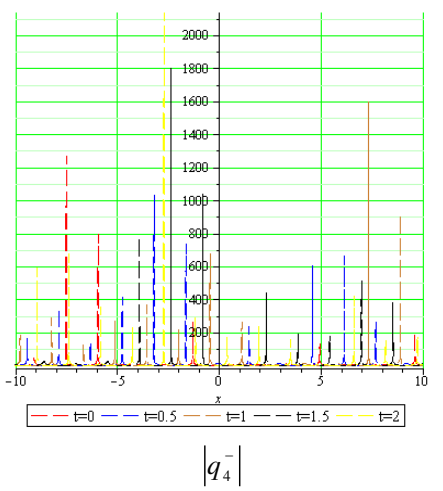

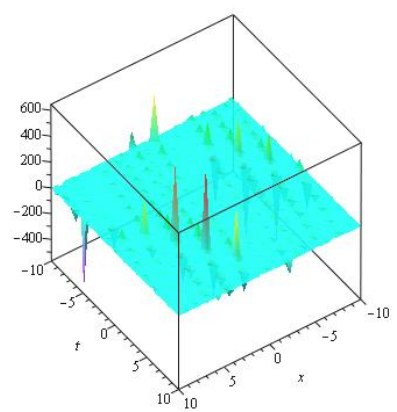

$\operatorname{Re}\left(q_{4}^{-}\right)$

(a)

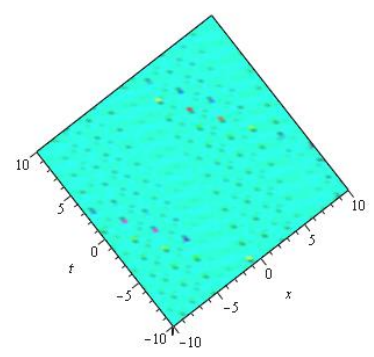

$\operatorname{Re}\left(q_{4}^{-}\right)$

(b)

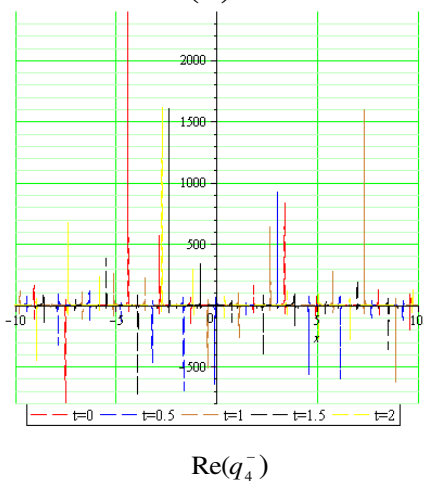

(c)

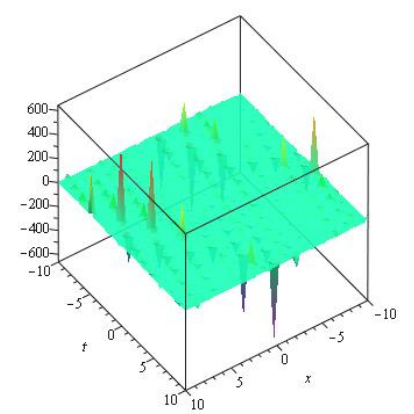

$\operatorname{Im}\left(q_{4}^{-}\right)$

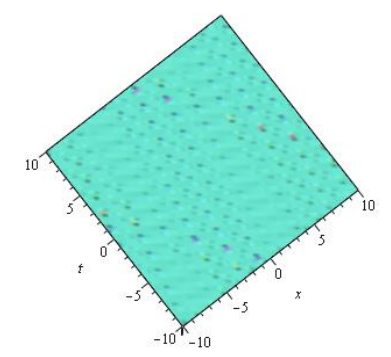

$\operatorname{Im}\left(q_{4}^{-}\right)$

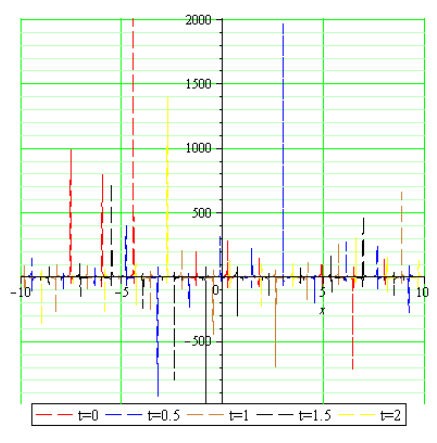

$\operatorname{Im}\left(q_{4}^{-}\right)$ 
Fig 2. (a) 3D-plot for $q_{4}^{-}$(b) the contour plot for $q_{4}^{-}$(c) 2D-plot for $q_{4}^{-}$at $\mathrm{t}=0, \mathrm{t}=0.5, \mathrm{t}=1, \mathrm{t}=1.5, \mathrm{t}=2$ .respectively, when $\omega=-4, \alpha=1, \vartheta_{1}=0.5, \vartheta_{2}=1, \beta=2, \chi_{1}=1.75, \chi_{2}=1.5, \eta_{1}=1, \eta_{2}=0.5, \varpi=2, \theta_{0}=0$ and $y=1.5$.

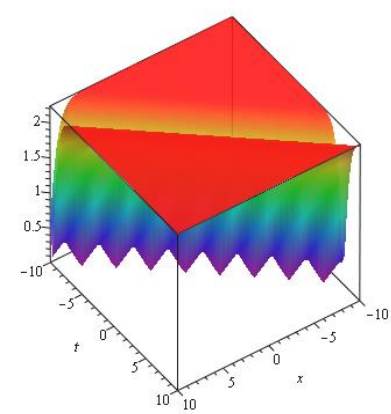

$\left|q_{5}^{+}\right|$

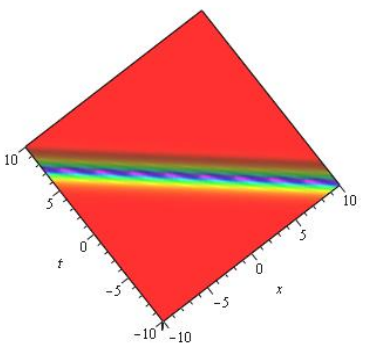

$\left|q_{5}^{+}\right|$

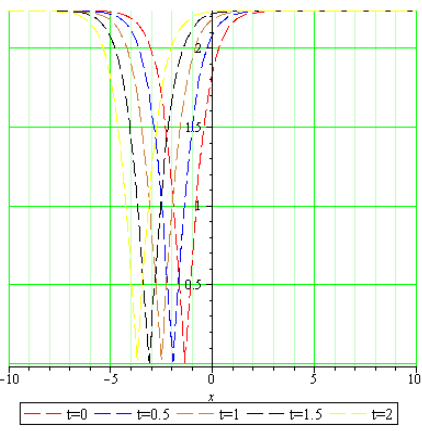

$\left|q_{5}^{+}\right|$

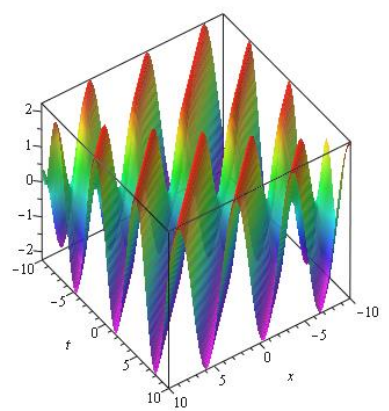

$\operatorname{Re}\left(q_{5}^{+}\right)$

(a)

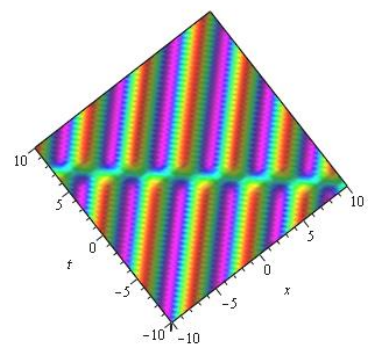

$\operatorname{Re}\left(q_{5}^{+}\right)$

(b)

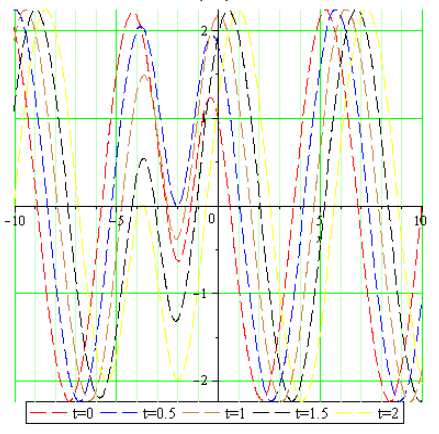

$\operatorname{Re}\left(q_{5}^{+}\right)$

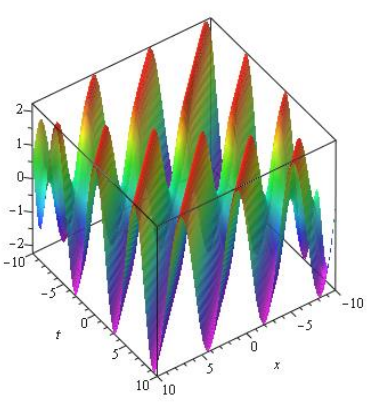

$\operatorname{Im}\left(q_{5}^{+}\right)$

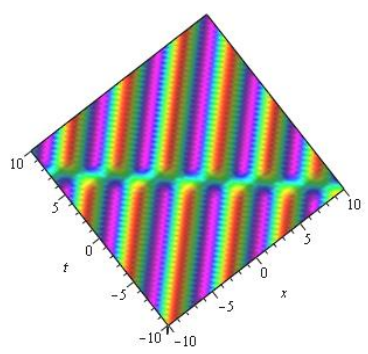

$\operatorname{Im}\left(q_{5}^{+}\right)$

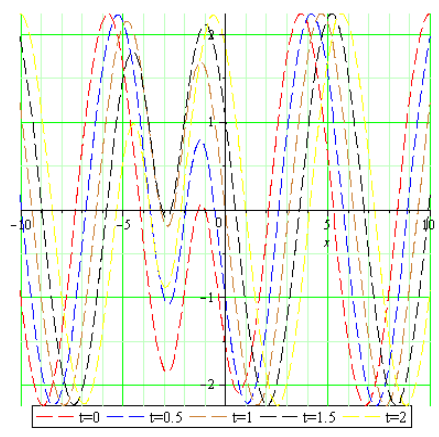

$\operatorname{Im}\left(q_{5}^{+}\right)$

(c)

Fig 3. (a) 3D-plot for $q_{1}^{+}$(b) the contour plot for $q_{1}^{+}$(c) 2D-plot for $q_{1}^{+}$at $\mathrm{t}=0, \mathrm{t}=0.5, \mathrm{t}=1, \mathrm{t}=1.5, \mathrm{t}=2$ .respectively, when $\omega=-2, \alpha=1, \vartheta_{1}=1, \vartheta_{2}=1, \beta=0.1, \chi_{1}=1.5, \chi_{2}=1, \eta_{1}=0.75, \eta_{2}=1, \varpi=1, \theta_{0}=1$ and $y=2$.

\section{Conclusion}

In this article functional variable method is applied to KMN model successfully to get the wave solutions of considered model. This model was first suggested not only to express the oceanic rogue waves but also to model the optical fiber communication. The solutions show that considered method fit well for nonlinear KMN model with complex structure.

\section{References}

[1] Pettersson, P., A. Doostan, and J. Nordstrom, Level set methods for stochastic 
discontinuity detection in nonlinear problems. Journal of Computational Physics, 2019. 392: p. 511-531.

[2] Liu, J., et al., Abundant exact solutions for the higher order non-linear Schrodinger equation with cubic-quintic non-Kerr terms. Communications in Nonlinear Science and Numerical Simulation, 2010. 15(12): p. 3777-3781.

[3] Kundu, A., A. Mukherjee, and T. Naskar, Modelling rogue waves through exact dynamical lump soliton controlled by ocean currents. Proceedings of the Royal Society a-Mathematical Physical and Engineering Sciences, 2014. 470(2164): p. 20.

[4] Rezazadeh, H., Korkmaz, A., Eslami, M., \&Mirhosseini-Alizamini, S.M. (2019). A large family of optical solutions to Kundu-Eckhaus model by a new auxiliary equation method. Optical and Quantum Electronics, 51(3), 84.

[5] Rezazadeh, H. (2018). New solitons solutions of the complex Ginzburg-Landau equation with Kerr law nonlinearity. Optik, 167, 218-227.

[6] Rezazadeh, H., Mirhosseini-Alizamini, S. M., Eslami, M., Rezazadeh, M., Mirzazadeh, M., \&Abbagari, S. (2018).New optical solitons of nonlinear conformable fractional Schrödinger-Hirota equation.Optik, 172, 545-553.

[7] Gao, W., Rezazadeh, H., Pinar, Z., Baskonus, H. M., Sarwar, S., \& Yel, G. (2020). Novel explicit solutions for the nonlinear Zoomeron equation by using newly extended direct algebraic technique. Optical and Quantum Electronics, 52(1), 1-13.

[8] Rezazadehd, H., Mirzazadeh, M., Mirhosseini-Alizamini, S.M., Neirameh, A., Eslami, M., \& Zhou, Q. (2018). Optical solitons of Lakshmanan-Porsezian-Daniel model with a couple of nonlinearities, Optik, 164, 414-423.

[9] Raza, N., Aslam, M. R., \&Rezazadeh, H. (2019). Analytical study of resonant optical solitons with variable coefficients in Kerr and non-Kerr law media.Optical and Quantum Electronics, 51(2), 59.

[10] Raza, N., Afzal, U., Butt, A. R., \&Rezazadeh, H. (2019). Optical solitons in nematic liquid crystals with Kerr and parabolic law nonlinearities.Optical and Quantum Electronics, 51(4), 107.

[11] Tasbozan, O., Kurt, A., \& Tozar, A. (2019). New optical solutions of complex GinzburgLandau equation arising in semiconductor lasers. Applied Physics B, 125(6), 104.

[12] Atilgan, E., Senol, M., Kurt, A., \& Tasbozan, O. (2019). New wave solutions of timefractional coupled Boussinesq-Whitham-Broer-Kaup equation as a model of water waves. China Ocean Engineering, 33(4), 477-483.

[13] Kurt, A. (2019). New periodic wave solutions of a time fractional integrable shallow water equation. Applied Ocean Research, 85, 128-135.

[14] Kurt, A., Tasbozan, O., \& Baleanu, D. (2017). New solutions for conformable fractional Nizhnik-Novikov-Veselov system via $\left(\mathrm{G}^{\prime} / \mathrm{G}\right)$-expansion method and homotopy analysis methods. Optical and Quantum Electronics, 49(10), 333.

[15] Seadawy, A. R., Ali, K. K., \& Nuruddeen, R. I. (2019). A variety of soliton solutions for the fractional Wazwaz-Benjamin-Bona-Mahony equations. Results in Physics, 12, 22342241.

[16] Ali, K. K., Nuruddeen, R. I., \& Hadhoud, A. R. (2018). New exact solitary wave solutions for the extended (3+1)-dimensional Jimbo-Miwa equations. Results in Physics, 9, 12-16.

[17] Ali, K. K., Nuruddeen, R. I., \& Raslan, K. R. (2018). New hyperbolic structures for the conformable time-fractional variant bussinesq equations. Optical and Quantum Electronics, 50(2), 61. 
[18] Raslan, K. R., El-Danaf, T. S., \& Ali, K. K. (2017). Exact solution of the space-time fractional coupled EW and coupled MEW equations. The European Physical Journal Plus, 132(7), 319.

[19] Seadawy, A. R., Nuruddeen, R. I., Aboodh, K. S., \& Zakariya, Y. F. (2020). On the exponential solutions to three extracts from extended fifth-order KdV equation. Journal of King Saud University-Science, 32(1), 765-769.

[20] Sedeeg, A. K. H., Nuruddeen, R. I., \& Gómez-Aguilar, J. F. (2019). Generalized optical soliton solutions to the (3+1)-dimensional resonant nonlinear Schrödinger equation with Kerr and parabolic law nonlinearities. Optical and Quantum Electronics, 51(6), 173.

[21] Sulaiman, T. A., Nuruddeen, R. I., \& Mikail, B. B. (2019). Dark and singular solitons to the two nonlinear Schrödinger equations. Optik, 186, 423-430.

[22] Sulaiman, T. A., \& Bulut, H. (2020). Optical solitons and modulation instability analysis of the (1+1)-dimensional coupled nonlinear Schrödinger equation. Communications in Theoretical Physics, 72(2), 025003.

[23] Sulaiman, T. A., Bulut, H., \& Atas, S. S. (2019). Optical solitons to the fractional Schrödinger-Hirota equation. Applied Mathematics and Nonlinear Sciences, 4(2), 535542.

[24] Sulaiman, T. A., \& Bulut, H. (2019). The solitary wave solutions to the fractional Radhakrishnan-Kundu-Lakshmanan model. International Journal of Modern Physics B, 33(31), 1950370.

[25] Liu, L., Tian, B., Wu, X. Y., \& Sun, Y. (2018). Higher-order rogue wave-like solutions for a nonautonomous nonlinear Schrödinger equation with external potentials.Physica A: Statistical Mechanics and its Applications, 492, 524-533.

[26] El-Dessoky, M. M., \& Islam, S. (2019). Resonant optical solitons of nonlinear Schrödinger equation with dual power law nonlinearity.Physica A: Statistical Mechanics and its Applications, 122445.

[27] Seadawy, A. R., \&Cheemaa, N. (2019). Propagation of nonlinear complex waves for the coupled nonlinear Schrödinger Equations in two core optical fibers. Physica A: Statistical Mechanics and its Applications, 121330.

[28] Zhang, Z. Y., Gan, X. Y., \& Yu, D. M. (2011). Bifurcation behaviour of the travelling wave solutions of the perturbed nonlinear Schrödinger equation with Kerr law nonlinearity.ZeitschriftfürNaturforschung A, 66(12), 721-727.

[29] Zhang, Z. Y., Li, Y. X., Liu, Z. H., \& Miao, X. J. (2011). New exact solutions to the perturbed nonlinear Schrödinger's equation with Kerr law nonlinearity via modified trigonometric function series method.Communications in Nonlinear Science and Numerical Simulation, 16(8), 3097-3106.

[30] Zhang, W., \&Simos, T. E. (2016). A high-order two-step phase-fitted method for the numerical solution of the Schrödinger equation.Mediterranean Journal of Mathematics, 13(6), 5177-5194.

[31] Zhang, L., \&Simos, T. E. (2016). An efficient numerical method for the solution of the Schrödinger equation.Advances in Mathematical Physics, 2016.

[32] Zerarka, A., \& Ouamane, S. (2010). Application of the functional variable method to a class of nonlinear wave equations. World Journal of Modelling and Simulation, 6(2), 150-160.

[33] Eslami, M., Rezazadeh, H., Rezazadeh, M., \& Mosavi, S. S. (2017). Exact solutions to the space-time fractional Schrödinger-Hirota equation and the space-time modified KDV-Zakharov-Kuznetsov equation. Optical and Quantum Electronics, 49(8), 279. 
[34] Bekir, A., Güner, Ö., Aksoy, E., \& Pandır, Y. (2015, March). Functional variable method for the nonlinear fractional differential equations. In AIP Conference Proceedings (Vol. 1648, No. 1, p. 730001). AIP Publishing LLC.

[35] Yildirım, Y. (2019). Optical solitons to Kundu-Mukherjee-Naskar model in birefringent fibers with trial equation approach. Optik, 183, 1026-1031.

[36] Rizvi, S. T. R., Afzal, I., \& Ali, K. (2020). Dark and singular optical solitons for KunduMukherjee-Naskar model. Modern Physics Letters B, 34(06), 2050074.

[37] Talarposhti, R. A., Jalili, P., Rezazadeh, H., Jalili, B., Ganji, D. D., Adel, W., \& Bekir, A. (2020). Optical soliton solutions to the $(2+1)$-dimensional Kundu-MukherjeeNaskar equation. International Journal of Modern Physics B, 34(11), 2050102. 


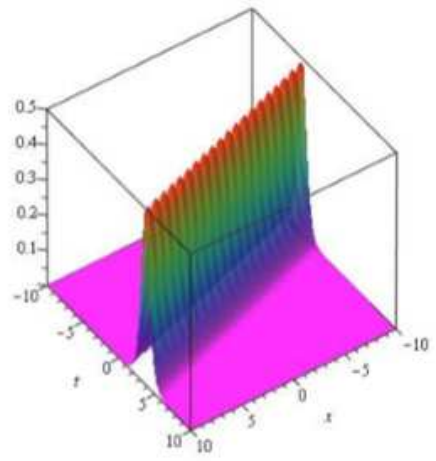

$\left|q_{1}^{+}\right|$

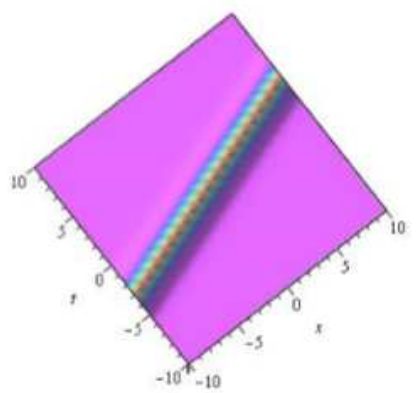

$\left|q_{1}^{+}\right|$

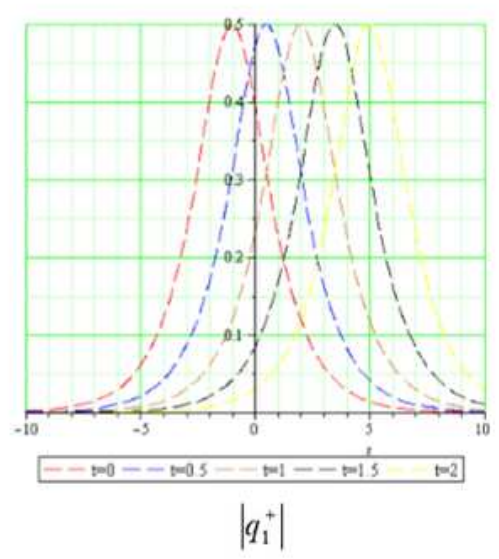

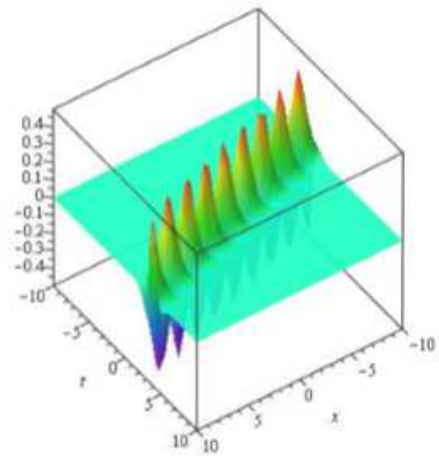

$\operatorname{Re}\left(q_{1}^{+}\right)$

(a)

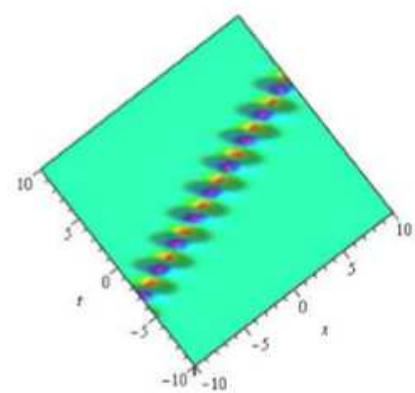

$\operatorname{Re}\left(q_{1}^{+}\right)$

(b)

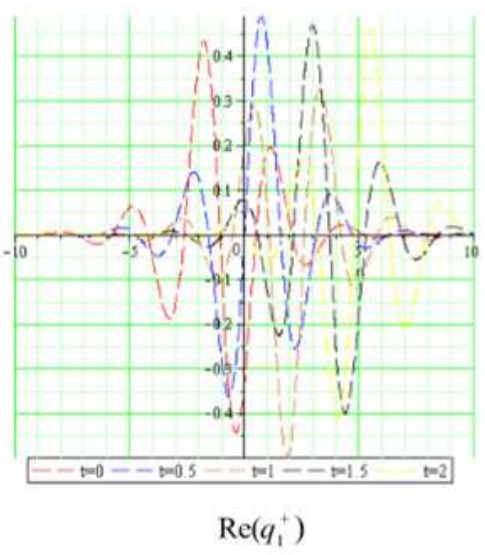

(c)

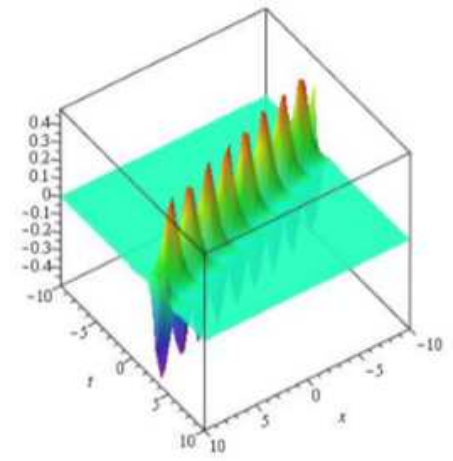

$\operatorname{Im}\left(q_{1}^{+}\right)$

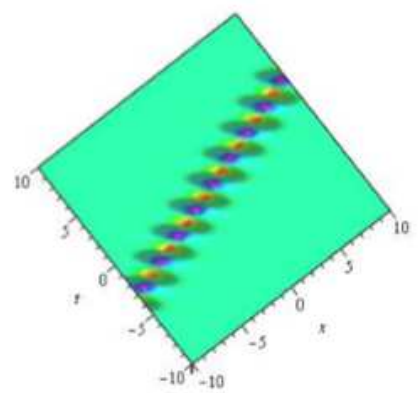

$\operatorname{Im}\left(q_{1}^{+}\right)$

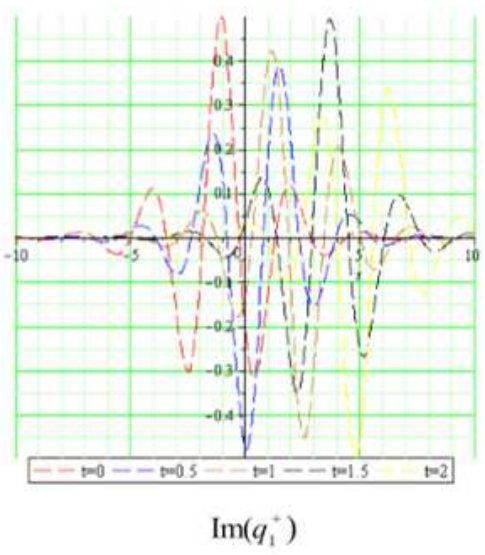

\section{Figure 1}

Please see the Manuscript PDF file for the complete figure caption. 


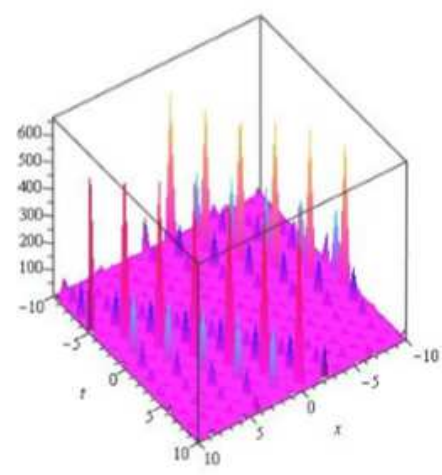

$\left|q_{4}^{-}\right|$

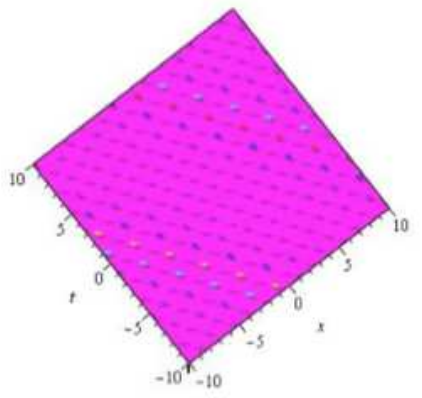

$\left|q_{4}^{-}\right|$

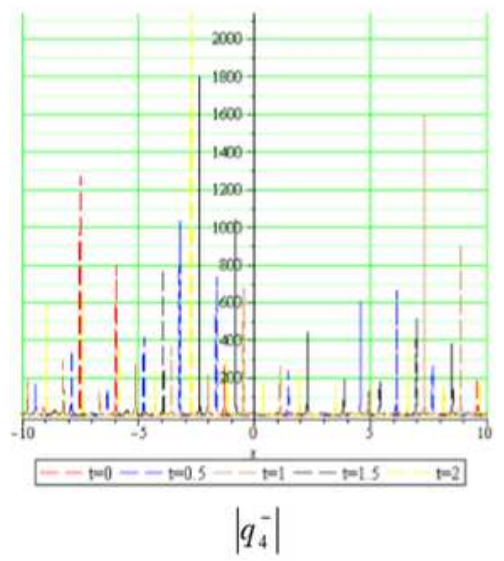

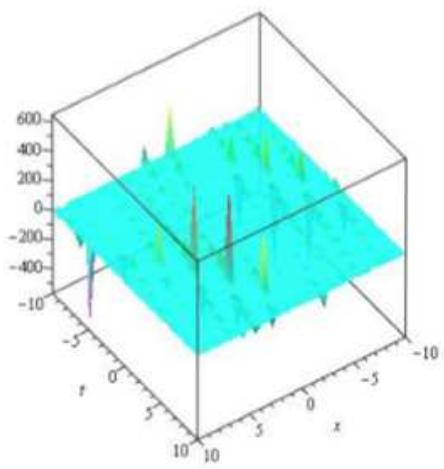

$\operatorname{Re}\left(q_{4}^{-}\right)$

(a)

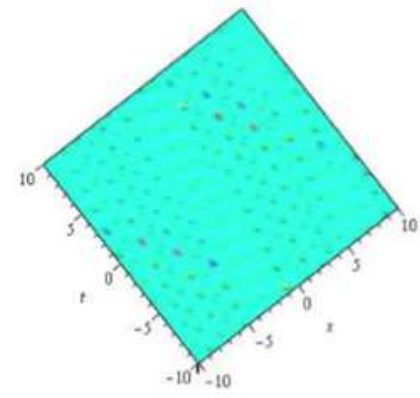

$\operatorname{Re}\left(q_{4}^{-}\right)$

(b)

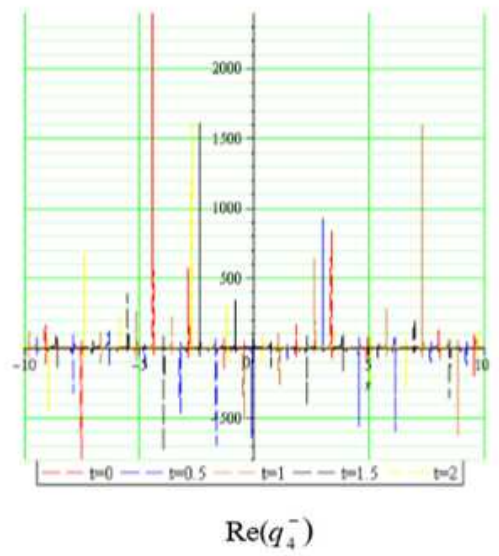

(c)

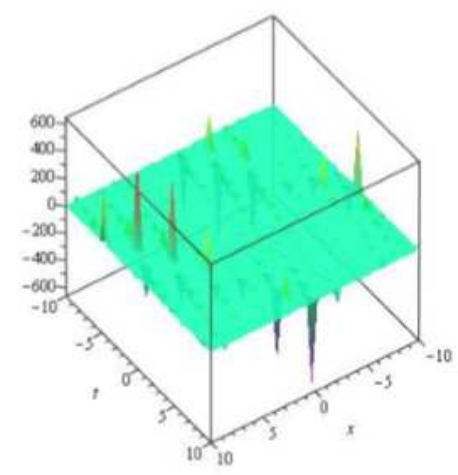

$\operatorname{Im}\left(q_{4}^{-}\right)$

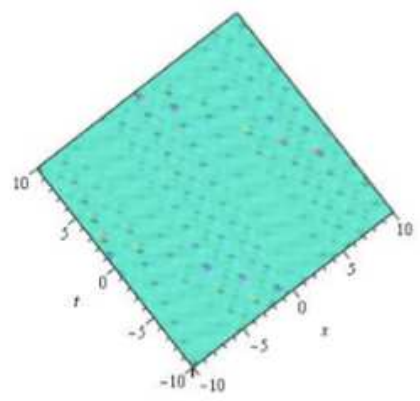

$\operatorname{Im}\left(q_{4}^{-}\right)$

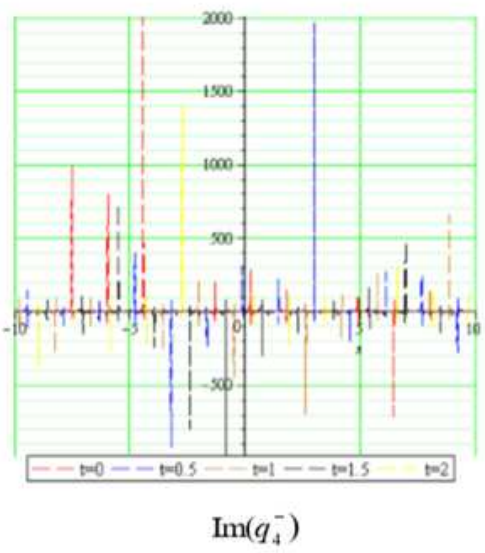

\section{Figure 2}

Please see the Manuscript PDF file for the complete figure caption. 


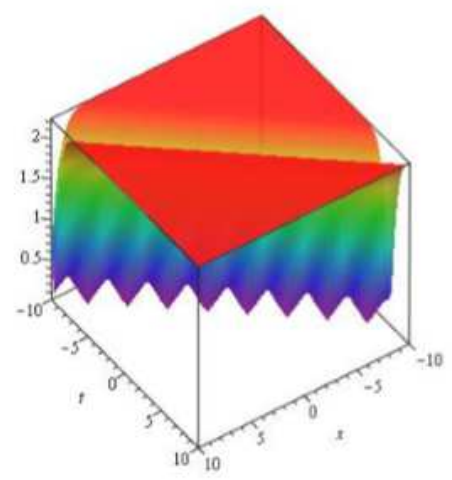

$\left|q_{s}^{+}\right|$

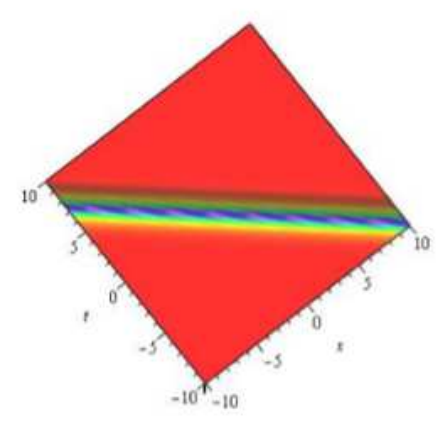

$\left|q_{s}^{+}\right|$

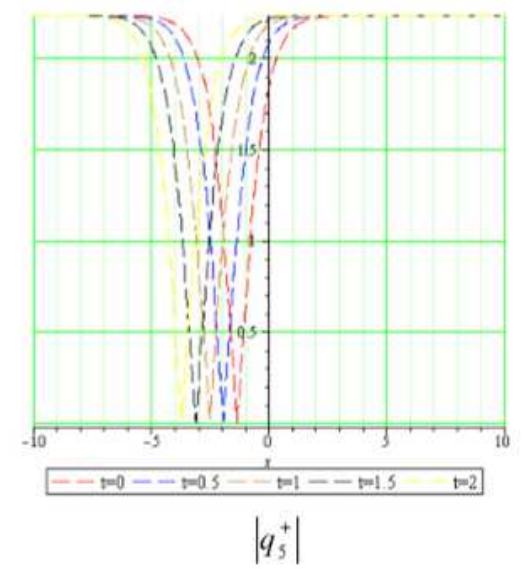

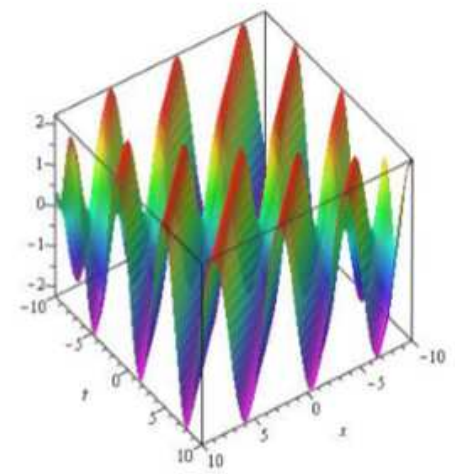

$\operatorname{Re}\left(q_{5}^{+}\right)$

(a)

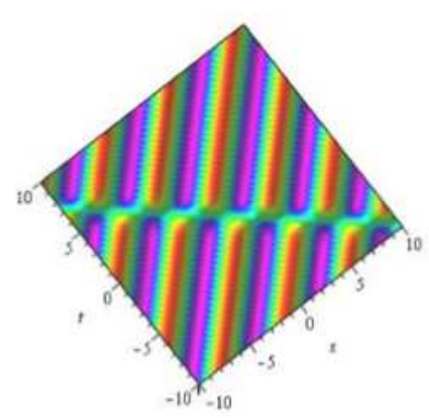

$\operatorname{Re}\left(q_{5}^{+}\right)$

(b)

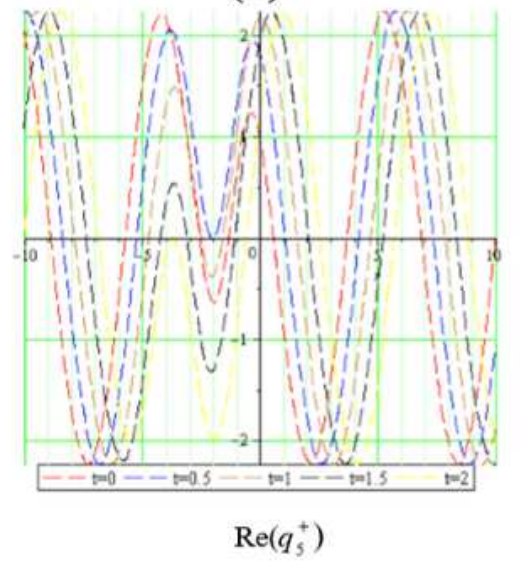

(c)

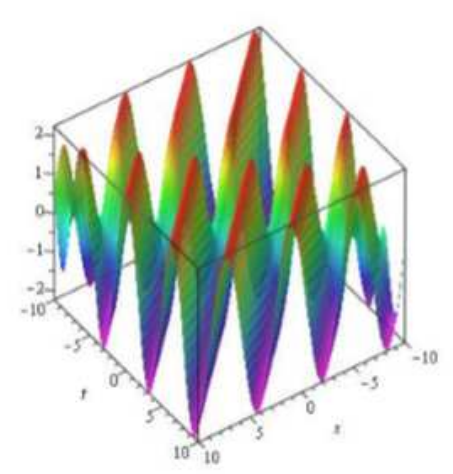

$\operatorname{Im}\left(q_{5}^{+}\right)$

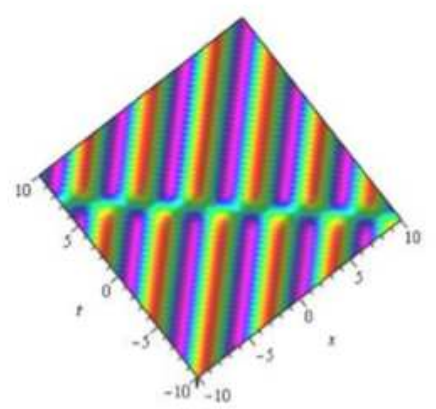

$\operatorname{Im}\left(q_{5}^{+}\right)$

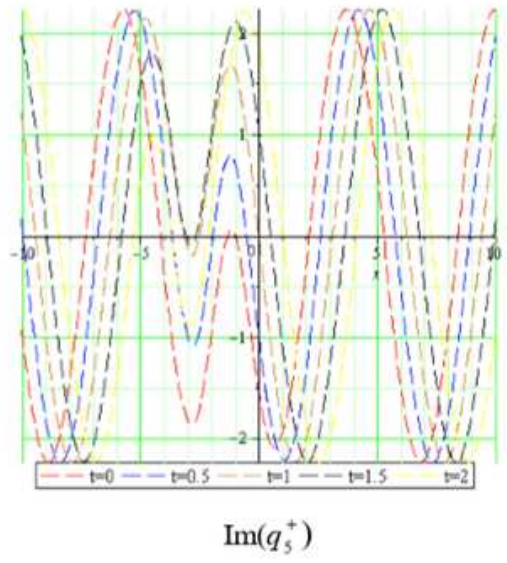

\section{Figure 3}

Please see the Manuscript PDF file for the complete figure caption. 